Authors

Feng-Lei Hong, Jun Ye, Long-Sheng Ma, Susanne Picard, Christian J. Bordé, and John L. Hall 


\title{
Rotation dependence of electric quadrupole hyperfine interaction in the ground state of molecular iodine by high-resolution laser spectroscopy
}

\author{
Feng-Lei Hong, ${ }^{*}$ Jun Ye, ${ }^{* *}$ Long-Sheng Ma, ${ }^{\dagger}$ Susanne Picard, ${ }^{\ddagger}$ Christian J. Bordé, ${ }^{\mathbb{I}}$ and John L. Hall** \\ JILA, National Institute of Standards and Technology, and University of Colorado, Boulder, Colorado 80309-
} 0440

Received June 12, 2000; revised manuscript received October 17, 2000

\begin{abstract}
Doppler-free high-resolution spectroscopy is applied to molecular iodine at $532 \mathrm{~nm}$ by Nd:YAG lasers. The main hyperfine components as well as the crossover lines are measured for $R(56) 32-0$ and $P(54) 32-0$ transitions by heterodyne beating of two $\mathrm{I}_{2}$-stabilized lasers. The measured hyperfine splittings including both main and crossover lines are fitted to a four-term Hamiltonian, which includes the electric quadrupole, spinrotation, tensor spin-spin, and scalar spin-spin interactions, with an average deviation of $\sim 1 \mathrm{kHz}$. Absolute values of the electric quadrupole hyperfine constants for both the upper and the lower states are obtained. The rotation dependence of the ground-state $\left(v^{\prime \prime}=0\right)$ electric quadrupole constant $e Q q^{\prime \prime}$ is found to be $e Q q^{\prime \prime}(J)=-2452.556(2)-0.000164(5) J(J+1)-0.000000005(2) J^{2}(J+1)^{2} \mathrm{MHz}$. (C) 2001 Optical Society of America

OCIS codes: $020.2930,300.6320,300.6390,120.3940,300.6460$.
\end{abstract}

\section{INTRODUCTION}

Molecular iodine $\left(\mathrm{I}_{2}\right)$ has always been an attractive subject for spectroscopy because of its rich spectra from the UV to the IR. Hyperfine interactions in $\mathrm{I}_{2}$ have been studied extensively in the past two decades, yielding increasing resolution and accuracy. The hyperfine splitting of iodine lines has been measured by Doppler-free laser spectroscopy with argon-ion lasers, ${ }^{1-3}$ krypton-ion lasers, ${ }^{1} \mathrm{He}-\mathrm{Ne}$ lasers, ${ }^{4-6}$ and dye lasers. ${ }^{7}$ A tabulation of the hyperfine structures in ${ }^{127} \mathrm{I}_{2}$ can be found in Ref. 8 . Recent research has increased the accuracy with which iodine can serve as wavelength and frequency references.

Recently, diode-pumped Nd:YAG lasers were recognized as promising sources for high-resolution spectroscopy because of their inherently low-frequency noise, high power, and high reliability. By locking of secondharmonic generation (SHG) of the Nd:YAG lasers to hyperfine lines of $\mathrm{I}_{2}$ at $532 \mathrm{~nm}$, lasers at JILA have reached the Allan frequency stability of $5 \times 10^{-14}$ at $1 \mathrm{~s}$, improving after $100 \mathrm{~s}$ to $\sim 5 \times 10^{-15} \cdot{ }^{9,13}$ The hyperfine splittings of iodine lines near $532 \mathrm{~nm}$ have been measured at Stanford University ${ }^{10,11}$ and at JILA. ${ }^{12,13}$

The hyperfine spectrum of iodine is important for metrological applications. One of the most popular ways to establish a realization of the Metre is to use a 633-nm $\mathrm{He}-\mathrm{Ne}$ laser that is frequency locked onto one of the hyperfine lines of $\mathrm{I}_{2}$. Furthermore, six of the twelve radiation values recommended for realization of the Metre are obtained from iodine-stabilized lasers. ${ }^{14}$ The absolute optical frequency of hyperfine component $a_{10}$ in the $R$ (56)32-0 transition at $532 \mathrm{~nm}$ has been measured at JILA, ${ }^{9,15,16}$ and frequency comparisons of $\mathrm{I}_{2}$-stabilized $\mathrm{Nd}$ :YAG lasers have been carried out internationally. ${ }^{17,18}$
$\mathrm{I}_{2}$-stabilized lasers are also used as frequency standards for precision spectroscopic measurements and accurate determination of physical constants.

$\mathrm{I}_{2}$ also provides a good subject with which to test theoretical models of hyperfine interactions. The Hamiltonian of the hyperfine interactions, $H_{\mathrm{hfs}}$, can be written $\mathrm{as}^{2,3}$

$$
\begin{aligned}
H_{\mathrm{hfs}}= & e Q q \times H_{\mathrm{EQ}}+C \times H_{\mathrm{SR}}+d \times H_{\mathrm{TSS}} \\
& +\delta \times H_{\mathrm{SSS}},
\end{aligned}
$$

where $H_{\mathrm{EQ}}, H_{\mathrm{SR}}, H_{\mathrm{TSS}}$, and $H_{\mathrm{SSS}}$ represent, respectively, the electric quadrupole, spin-rotation, tensor spin-spin, and scalar spin-spin interactions and $e Q q, C, d$, and $\delta$ represent the corresponding hyperfine constant for each of these interactions. Because the selection rules of the main rovibrational transitions between the $X$ (ground) and the $B$ (excited) electronic states are very strict $(\Delta F$ $=\Delta J, \Delta J= \pm 1)$, the hyperfine splitting patterns are nearly identical to those of either the initial or the final state, except that the splittings are scaled by the differences between the hyperfine constants in the two states. In other words, in these main transitions the hyperfine structure quantum numbers remain unchanged, whereas only the hyperfine coupling constants change. The differences in the hyperfine constants between the excited and the ground states are written as

$$
\begin{aligned}
\Delta(e Q q) & =e Q q^{\prime}-e Q q^{\prime \prime}, \\
\Delta C & =C^{\prime}-C^{\prime \prime}, \\
\Delta d & =d^{\prime}-d^{\prime \prime}, \\
\Delta \delta & =\delta^{\prime}-\delta^{\prime \prime} .
\end{aligned}
$$


By fitting the observed spectra of the main transitions to the theoretical expectations represented by Eq. (1), one can obtain accurate values for $\Delta(e Q q), \Delta C, \Delta d$, and $\Delta \delta$ but only a crude estimate of the absolute values for the respective ground and excited states. However, by observing also the so-called crossover transitions between the main lines $(\Delta F=\Delta J)$ and the much weaker forbidden transitions $(\Delta F=0)$, one can determine accurately the absolute values of the hyperfine constants for both the upper and the lower states. In the pioneering research, the ground state $e Q q^{\prime \prime}$ for $v^{\prime \prime}=0$ and $J^{\prime \prime}=13$ was measured by this method and found to be $-2452 \pm 40 \mathrm{MHz} .{ }^{19}$

Using measurements made by the molecular-beam magnetic resonance method, Yokozeki and Muenter measured and calculated the four hyperfine constants of the ground state $\left(v^{\prime \prime}=0\right.$ and $\left.J^{\prime \prime}=13\right)$ with good precision. $^{20}$ Their values, $e Q q^{\prime \prime}=-2452583.7(16) \mathrm{kHz}, C^{\prime \prime}$ $=3.162(8) \mathrm{kHz}, d^{\prime \prime}=1.58(5) \mathrm{kHz}$, and $\delta^{\prime \prime}=3.66(3)$ $\mathrm{kHz}$, have been used in many subsequent calculations ${ }^{10-13}$ to represent the lower state and thus to allow one to obtain the hyperfine constants for the excited states. (Here we use the convention that the standard uncertainty in parentheses applies to the last digit of the value.) However, the quoted hyperfine-constant values give only a close approximation in those calculations because different rotational states are involved, albeit in the same vibrational ground state. The question is: Is there measurable rotational dependence for those hyperfine constants of the ground state with $v^{\prime \prime}=0$ ? Recently, Bordé and his colleagues ${ }^{21,22}$ measured the hyperfine splittings in the ground state for $J^{\prime \prime}=13$ and $J^{\prime \prime}=15$ rotational levels by using stimulated Raman spectroscopy and derived hyperfine constants with higher accuracy than those described in Ref. 20. Within the experimental precision, they found that the constants were identical for both rotational levels, except for quadrupole coupling constants $e Q q^{\prime \prime}$, which differed from $J^{\prime \prime}=13$ and $J^{\prime \prime}=15$.

We measured both main and crossover lines for the $R$ (56)32-0 and P(54)32-0 transitions by using modulation transfer spectroscopy. ${ }^{23-25}$ Both main and crossover lines were fitted to the four-term Hamiltonian as shown in Eq. (1). From this, absolute values of the hyperfine constants of the upper and lower levels for both $J^{\prime \prime}=56$ and $J^{\prime \prime}=54$ transitions were derived.

With the high signal-to-noise $(\mathrm{S} / \mathrm{N})$ ratio achieved in the JILA iodine spectrometer and the high stability and accuracy attained as a consequence, ${ }^{9,12,13}$ we could fit the observed results including both main and crossover lines to the four-term Hamiltonian with $\sim 1-\mathrm{kHz}$ uncertainty. From the fitting, we could also observe various $e Q q^{\prime \prime}$ values for the ground states with different $J$ numbers. Combining our values with the $e Q q^{\prime \prime}$ values from Ref. 21, we obtained a formula for the rotation dependence of the ground state $e Q q^{\prime \prime}$.

\section{EXPERIMENT}

\section{A. Experimental Setup}

Figure 1 shows a block diagram of our measurement system. The optical part of the system contains a source laser oscillator, a buildup cavity for SHG, and an iodine spectrometer. The source oscillator of each system is a

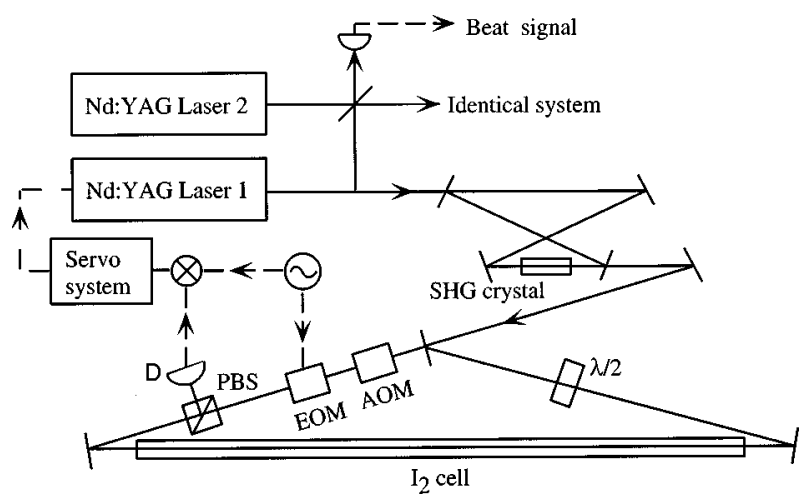

Fig. 1. Diagram of the experimental setup: D, detector; otherabbreviations defined in text.

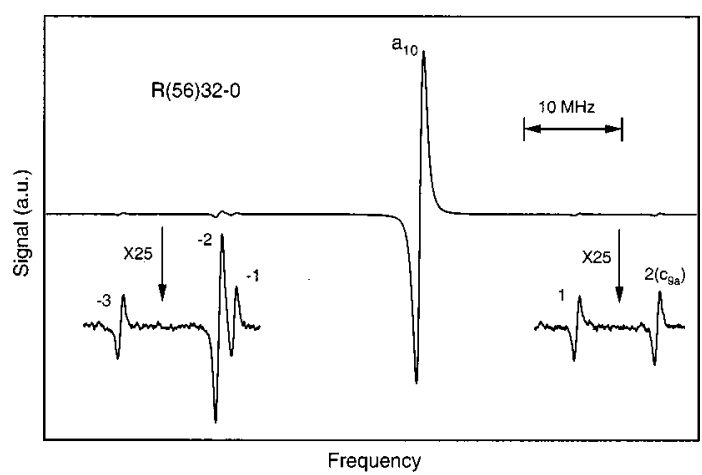

Fig. 2. Observed main hyperfine component $a_{10}$ of the $R$ (56)32-0 transition and the crossover lines nearby. The crossover lines are numbered in series as $-1,-2,-3, \ldots$ if the frequency is lower than that of the $a_{10}$ component and as $1,2, \ldots$ if the frequency is higher than that of $a_{10}$. Crossover line 2, numbered $c_{9 a}$, is included in the calculation.

commercial diode-pumped Nd:YAG laser, which has a nonplanar ring resonator formed in the monolithic gain medium. SHG of each system is accomplished through an external ring buildup cavity with an intracavity nonlinear crystal, either $\mathrm{KNbO}_{3}$ or $\mathrm{MgO}: \mathrm{LiNbO}_{3}$. The SHG cavities are locked to the source oscillators by either a dither method ${ }^{12}$ or a polarization method. ${ }^{23}$

The spectroscopy of molecular iodine is based on the sub-Doppler technique of modulation transfer, ${ }^{24-26}$ which gives a nearly flat baseline and is therefore quite attractive for laser spectroscopy and frequency stabilization. As shown in Fig. 1, the green beam from the SHG cavity is divided, by an appropriate beam splitter, into a strong pump beam and a weak probe beam in the iodine spectrometer. The pump beam is frequency shifted by an acousto-optic modulator (AOM) and is phase modulated by an electro-optic modulator (EOM). The AOM works as an optical isolator to prevent interferometric noise in the spectrometer. To further reduce the interference between the linearly polarized pump and probe beams, we rotate the polarization of the probe beam with a $\lambda / 2$ plate to be orthogonal to that of the pump beam. The probe beam, separated by a polarization beam splitter (PBS), reaches a detector after interacting with the pump beam inside the 1.2-m-long iodine cell. The unmodulated probe beam passes through the iodine cell and, as a result of nonlinear four-wave mixing with the modulated pump 
beam, develops new sidebands. Beat currents at the modulation frequency are generated in the photodetector between the new sidebands and the laser carrier. This signal is demodulated by a double-balanced mixer and used to control the laser frequency through a servo system.

Two independent systems were built in which each laser frequency is doubled and locked to its own iodine cell. The heterodyne beat frequency between the two lasers is measured at $1064 \mathrm{~nm}$ (IR light) by an avalanche photodetector. All the measured frequency intervals (in the IR) described in this paper have been multiplied by a factor of 2 , corresponding to correct interval values at $532 \mathrm{~nm}$. More-detailed descriptions of the systems are given elsewhere. ${ }^{12,13}$

\section{B. Observation of Main and Crossover Hyperfine Transitions}

Figure 2 shows the observed modulation transfer signal of the $a_{10}$ hyperfine component of the $R(56) 32-0$ transition and the crossover lines nearby. The EOM worked at a modulation frequency of $350 \mathrm{kHz}$ with a modulation index of $\sim 0.9$. The AOM was driven by an $80-\mathrm{MHz}$ rf source. The cold-finger temperature of the iodine cell was held at $-15{ }^{\circ} \mathrm{C}$, corresponding to an iodine-vapor pressure of $0.787 \mathrm{~Pa}$. The pump power was $7.6 \mathrm{~mW}$, and the probe power was $0.4 \mathrm{~mW}$. As discussed in Ref. 13, although the crossover resonances are $\sim 1 /(2 J)$ less intense than the main lines, they will increase in size much faster than the main lines when the input optical power is increased in the saturation spectrometer. This is so simply because the main lines have already been operated in the saturation regime, whereas the crossover can still grow linearly as a result of the unsaturated $\Delta F=0$ transitions.

We also present the crossover lines in Fig. 2 by expanding the signal size 25 times vertically. The $\mathrm{S} / \mathrm{N}$ ratio for the crossover lines is $\sim 100$ in a $32-\mathrm{Hz}$ bandwidth (5-ms time constant). The crossover lines are numbered in series here as $-1,-2,-3, \ldots$ if their frequencies are lower than that of the $a_{10}$ component and are numbered as 1 ,

Table 1. Observed and Calculated Hyperfine Components of $R(56) 32-0^{a}$

\begin{tabular}{|c|c|c|c|c|c|c|}
\hline $\begin{array}{l}\text { Hyperfine } \\
\text { Components }\end{array}$ & $\begin{array}{l}\text { Observed } \\
(\mathrm{kHz})\end{array}$ & $\begin{array}{l}\text { Calculated } \\
\quad(\mathrm{kHz})\end{array}$ & $\begin{array}{l}\text { Obs. - Cal. } \\
\quad(\mathrm{kHz})\end{array}$ & Weight & $\begin{array}{c}\text { Total } \\
\text { Angular } \\
\text { Momentum } \\
(F)\end{array}$ & $\begin{array}{c}\text { Total } \\
\text { Nuclear } \\
\text { Spin (I) }\end{array}$ \\
\hline \multicolumn{7}{|l|}{ Main } \\
\hline$a_{1}$ & 0 & 0 & 0 & 10.0 & 57 & 2 \\
\hline$a_{2}$ & 259697.4 & 259696.6 & 0.8 & 10.0 & 53 & 4 \\
\hline$a_{5}$ & 311366.8 & 311366.6 & 0.2 & 10.0 & 61 & 4 \\
\hline$a_{6}$ & 401477.5 & 401478.3 & -0.7 & 10.0 & 54 & 4 \\
\hline$a_{7}$ & 416993.1 & 416994.0 & -0.9 & 10.0 & 55 & 4 \\
\hline$a_{8}$ & 439626.5 & 439626.7 & -0.2 & 10.0 & 59 & 4 \\
\hline$a_{9}$ & 455343.2 & 455343.8 & -0.6 & 10.0 & 60 & 4 \\
\hline$a_{10}$ & 571541.8 & 571541.3 & 0.5 & 10.0 & 57 & 4 \\
\hline$a_{11}$ & 698054.2 & 698054.6 & -0.4 & 10.0 & 55 & 2 \\
\hline$a_{12}$ & 702754.1 & 702753.2 & 0.9 & 10.0 & 56 & 4 \\
\hline$a_{13}$ & 726030.5 & 726029.9 & 0.6 & 10.0 & 58 & 4 \\
\hline$a_{14}$ & 732207.1 & 732207.3 & -0.2 & 10.0 & 59 & 2 \\
\hline$a_{15}$ & 857953.7 & 857953.8 & -0.1 & 10.0 & 57 & 0 \\
\hline \multicolumn{5}{|l|}{ Crossover } & \multicolumn{2}{|c|}{ Common Level } \\
\hline$c_{1 a}$ & 180446.2 & 180448.5 & -2.3 & 1.0 & \multicolumn{2}{|c|}{ Upper } \\
\hline$c_{2 a}$ & 241268.5 & 241265.6 & 2.9 & 1.0 & \multicolumn{2}{|c|}{ Lower } \\
\hline$c_{3 a}$ & 329445.1 & 329448.2 & -3.1 & 1.0 & \multicolumn{2}{|c|}{ Upper } \\
\hline$c_{4 a}$ & 347630.6 & 347628.2 & 2.4 & 1.0 & \multicolumn{2}{|c|}{ Upper } \\
\hline$c_{5 a}$ & 359414.5 & 359416.6 & -2.1 & 1.0 & \multicolumn{2}{|c|}{ Upper } \\
\hline$c_{6 a}$ & 381364.7 & 381363.8 & 0.9 & 1.0 & \multicolumn{2}{|c|}{ Upper } \\
\hline$c_{7 a}$ & 384282.6 & 384281.4 & 1.2 & 1.0 & \multicolumn{2}{|c|}{ Upper } \\
\hline$c_{8 a}$ & 446451.4 & 446448.7 & 2.7 & 1.0 & \multicolumn{2}{|c|}{ Upper } \\
\hline$c_{9 a}$ & 597525.2 & 597526.0 & -0.8 & 1.0 & \multicolumn{2}{|c|}{ Upper } \\
\hline$c_{10 a}$ & 609179.0 & 609177.1 & 1.9 & 1.0 & \multicolumn{2}{|c|}{ Lower } \\
\hline$c_{11 a}$ & 614970.6 & 614973.0 & -2.4 & 1.0 & \multicolumn{2}{|c|}{ Upper } \\
\hline$c_{12 a}$ & 667603.5 & 667606.5 & -3.0 & 1.0 & \multicolumn{2}{|c|}{ Upper } \\
\hline$c_{13 a}$ & 769967.2 & 769968.0 & -0.8 & 1.0 & \multicolumn{2}{|c|}{ Upper } \\
\hline$c_{14 a}$ & 799024.4 & 799024.0 & 0.4 & 1.0 & \multicolumn{2}{|c|}{ Upper } \\
\hline$c_{15 a}$ & 876626.1 & 876624.3 & 1.8 & 1.0 & \multicolumn{2}{|c|}{ Lower } \\
\hline
\end{tabular}

${ }^{a}$ The standard deviation of the fit, including both main and crossover lines, is $0.95 \mathrm{kHz}$. 
Table 2. Observed and Calculated Hyperfine Components of $P(54) 32-0^{a}$

\begin{tabular}{|c|c|c|c|c|c|c|}
\hline $\begin{array}{l}\text { Hyperfine } \\
\text { Components }\end{array}$ & $\begin{array}{l}\text { Observed } \\
(\mathrm{kHz})\end{array}$ & $\begin{array}{l}\text { Calculated } \\
\quad(\mathrm{kHz})\end{array}$ & $\begin{array}{l}\text { Obs. - Cal. } \\
(\mathrm{kHz})\end{array}$ & Weight & $\begin{array}{c}\text { Total } \\
\text { Angular } \\
\text { Momentum }(F)\end{array}$ & $\begin{array}{l}\text { Total } \\
\text { Nuclear } \\
\text { Spin (I) }\end{array}$ \\
\hline \multicolumn{7}{|l|}{ Main } \\
\hline$a_{1}$ & 0 & 0 & 0 & 10.0 & 53 & 2 \\
\hline$a_{2}$ & $260992.4^{b}$ & 260992.0 & 0.4 & 10.0 & 49 & 4 \\
\hline$a_{3}$ & $285007.7^{b}$ & 285008.3 & -0.6 & 10.0 & 54 & 2 \\
\hline$a_{4}$ & $286726.4^{b}$ & 286726.1 & 0.3 & 10.0 & 52 & 2 \\
\hline$a_{5}$ & $310066.4^{b}$ & 310066.6 & -0.2 & 10.0 & 57 & 4 \\
\hline$a_{6}$ & $402249.5^{b}$ & 402250.2 & -0.7 & 10.0 & 50 & 4 \\
\hline$a_{7}$ & $417667.7^{b}$ & 417668.0 & -0.3 & 10.0 & 51 & 4 \\
\hline$a_{8}$ & $438918.6^{b}$ & 438918.2 & 0.4 & 10.0 & 55 & 4 \\
\hline$a_{9}$ & $454563.4^{b}$ & 454563.4 & 0.0 & 10.0 & 56 & 4 \\
\hline$a_{10}$ & $571536.4^{b}$ & 571535.6 & 0.8 & 10.0 & 53 & 4 \\
\hline$a_{11}$ & $698613.6^{b}$ & 698613.6 & 0.0 & 10.0 & 51 & 2 \\
\hline$a_{12}$ & $702934.8^{b}$ & 702934.5 & 0.3 & 10.0 & 52 & 4 \\
\hline$a_{13}$ & $725833.9^{b}$ & 725833.5 & 0.4 & 10.0 & 54 & 4 \\
\hline$a_{14}$ & $731687.9^{b}$ & 731688.0 & -0.1 & 10.0 & 55 & 2 \\
\hline$a_{15}$ & $857960.8^{b}$ & 857960.9 & -0.1 & 10.0 & 53 & 0 \\
\hline \multicolumn{5}{|l|}{ Crossover } & \multicolumn{2}{|c|}{ Common Level } \\
\hline$c_{1 a}$ & -37820.4 & -37827.0 & 6.6 & 1.0 & \multicolumn{2}{|c|}{ Lower } \\
\hline$c_{2 a}$ & 342911.6 & 342916.7 & -5.1 & 1.0 & \multicolumn{2}{|c|}{ Upper } \\
\hline$c_{3 a}$ & 374079.4 & 374077.8 & 1.6 & 1.0 & \multicolumn{2}{|c|}{ Upper } \\
\hline$c_{4 a}$ & 478368.0 & 478363.5 & 4.5 & 1.0 & \multicolumn{2}{|c|}{ Lower } \\
\hline$c_{5 a}$ & 629601.2 & 629605.8 & -4.6 & 1.0 & \multicolumn{2}{|c|}{ Upper } \\
\hline$c_{6 a}$ & 659140.7 & 659139.5 & 1.2 & 1.0 & \multicolumn{2}{|c|}{ Upper } \\
\hline$c_{7 a}$ & 813656.2 & 813659.2 & -3.0 & 1.0 & \multicolumn{2}{|c|}{ Upper } \\
\hline$c_{8 a}$ & 879721.1 & 879722.8 & -1.7 & 1.0 & \multicolumn{2}{|c|}{ Lower } \\
\hline
\end{tabular}

${ }^{a}$ The standard deviation of the fit including both main and crossover lines is $1.13 \mathrm{kHz}$.

${ }^{b}$ The frequency values of the observed main lines were published in Ref. 13.

Table 3. Fitted Hyperfine Constants

\begin{tabular}{lcccc}
\hline Parameter & $R(56) 32-0$ & $P(54) 32-0$ & $R(15) 43-0^{a}$ & $P(13) 43-0^{a}$ \\
\hline$e Q q^{\prime \prime}(\mathrm{MHz})$ & $-2453.132(11)$ & $-2453.088(24)$ & $-2452.59699(45)$ & $-2452.58514(45)$ \\
$C^{\prime \prime}(\mathrm{kHz})$ & $3.306(21)$ & $2.904(40)$ & $3.1543(29)$ & $1.519(18)$ \\
$d^{\prime \prime}(\mathrm{kHz})$ & $1.524^{b}$ & $1.524^{b}$ & $3.701(23)$ & $1.528(18)$ \\
$\delta^{\prime \prime}(\mathrm{kHz})$ & $3.705^{b}$ & $3.705^{b}$ & - & - \\
$e Q q^{\prime}(\mathrm{MHz})$ & $-544.751(11)$ & $-544.656(24)$ & - & - \\
$C^{\prime}(\mathrm{kHz})$ & $89.656(21)$ & $89.004(41)$ & - & - \\
$d^{\prime}(\mathrm{kHz})$ & $-42.701(78)$ & $-42.54(11)$ & - & - \\
$\delta^{\prime}(\mathrm{kHz})$ & $-6.943(86)$ & $-7.04(12)$ & - & - \\
$\Delta e Q q(\mathrm{MHz})$ & $1908.381(1)^{c}$ & $1908.432(1)^{c}$ & - & - \\
$\Delta C(\mathrm{kHz})$ & $86.350^{c}$ & $86.100^{c}$ & - & - \\
$\Delta d(\mathrm{kHz})$ & $-44.225^{c}$ & $-44.064^{c}$ & - & - \\
$\Delta \delta(\mathrm{kHz})$ & $-10.647^{c}$ & $-10.744^{c}$ & \\
\hline
\end{tabular}

${ }^{a}$ The hyperfine constants of $R(15) 43-0$ and $P(13) 43-0$ are taken from Refs. 21 and 22 .

${ }^{b} d^{\prime \prime}$ and $\delta^{\prime \prime}$ in the present fitting are fixed to the values in Refs. 21 and 22 .

${ }^{c}$ The uncertainty of the relative values of the hyperfine constants is much smaller than that of the absolute values. For example, the uncertainty of $\Delta e Q q$ of the $R$ (56)32-0 transition calculated with only main lines is $\sim 1 \mathrm{kHz}$, whereas the uncertainty of the absolute values of the upper and lower levels is $11 \mathrm{kHz}$.

$2, \ldots$ if their frequencies are higher than that of $a_{10} . \quad$ It is worth mentioning here that no crossover line was found near $a_{10}$, a contrary result that would have affected the line shape and the locking condition of the $a_{10}$ component (which is the one used for optical frequency standards).
The hyperfine splittings of both the main and the crossover lines were measured by heterodyne beating of the two iodine-stabilized Nd:YAG lasers. One laser was locked onto a reference line, and the other laser was locked onto those main and crossover lines to be mea- 
sured. To reduce the power broadening and the power shift for the main lines, we adjusted the pump (probe) power to $\sim 2.0(0.4) \mathrm{mW}$ for the main-line measurements.

The measured main and crossover lines of the $R(56) 32-0$ transition are listed in Table 1. For the measurement of main lines, the standard deviation of the beat frequency noise is $\sim 40 \mathrm{~Hz}$, corresponding to $\sim 28-\mathrm{Hz} \mathrm{rms}$ noise per laser at 1-s averaging time. The uncertainty of the measured main hyperfine lines, including all the differences between the two spectrometers (iodine cell, modulation frequency, beam size, intensity, etc.), is typically $\pm 300 \mathrm{~Hz}$. A detailed description of the stability and uncertainty of the main-line measurements is given in Ref. 13. For the measurement of crossover lines, the bigger uncertainty arises from the smaller $\mathrm{S} / \mathrm{N}$ ratio. The corresponding standard deviation of the typical beat frequency noise is $\sim 1 \mathrm{kHz}$ at 1-s averaging time. Some of the crossover lines are not included in the Hamiltonian calculation described in Section 3 below. For example, lines -1 and -2 near $a_{10}$ are excluded because they are too close to each other, and lines 1 and -3 are excluded because their measured standard deviation has exceeded $3 \mathrm{kHz}$. Crossover line 2, which is included in the calculation, is designated $c_{9 a}$.

The measured main and crossover lines of the $P$ (54)32-0 transition are listed in Table 2. The values of the main line splittings have been published in Ref. 13 .

\section{CALCULATION OF HYPERFINE SPLITTINGS AND COUPLING CONSTANTS}

As described in Section 1, the hyperfine Hamiltonian can be written as

$$
\begin{aligned}
H_{\mathrm{hfs}}= & e Q q \times H_{\mathrm{EQ}}+C \times H_{\mathrm{SR}}+d \times H_{\mathrm{TSS}} \\
& +\delta \times H_{\mathrm{SSS}} .
\end{aligned}
$$

The electric quadrupole interaction $e Q q \times H_{\mathrm{EQ}}$ and the spin-rotation interaction $C \times H_{\mathrm{SR}}$ were introduced by $\mathrm{Kroll}^{27}$; the tensor spin-spin interaction $d \times H_{\mathrm{TSS}}$ was introduced by Bunker and Hanes ${ }^{28}$; the scalar spin-spin interaction $\delta \times H_{\mathrm{SSS}}$ was introduced by Hackel et al. ${ }^{29,30}$ We follow the procedure outlined by Bordé et al. ${ }^{3}$ to calculate the eigenstates of the hyperfine Hamiltonian. In calculating the electric quadrupole interactions, we take $\Delta J$ up to \pm 4 into account. The rotational Hamiltonian $H_{R}$,

$$
\begin{aligned}
\left\langle J I F\left|H_{R}\right| J I F\right\rangle= & B J(J+1)-D J^{2}(J+1)^{2} \\
& +H J^{3}(J+1)^{3},
\end{aligned}
$$

was introduced in this calculation, where $I$ is the total nuclear spin and $F$ is the total angular momentum. The necessary rotational constants $B, D$, and $H$ are taken from Ref. 31. As our observed results include both main and crossover lines, not only the differences but also the absolute values of the hyperfine constants of both upper and lower levels can be obtained. In the present calculation, the hyperfine splittings are fitted to the measurements by a least-squares fit, where the hyperfine constants $e Q q^{\prime \prime}, e Q q^{\prime}, C^{\prime \prime}, C^{\prime}, d^{\prime}$, and $\delta^{\prime}$ are varied. The lower-level hyperfine constants $d^{\prime \prime}$ and $\delta^{\prime \prime}$ are fixed to the values given in Ref. 21 and shown in Table 3. This is reasonable because $d^{\prime \prime}$ and $\delta^{\prime \prime} \operatorname{did}$ not show $J$ dependence in Ref. 21, and we could obtain higher fitting accuracy by fixing $d^{\prime \prime}$ and $\delta^{\prime \prime}$. The lower-level hyperfine constant $C^{\prime \prime}$ is not fixed in the present calculation, because the observed intervals of the crossover lines could be better reproduced by the least-squares fit when $C^{\prime \prime}$ was also varied. A weight of 10 is applied to the measured main lines; a weight of 1 is applied to the measured crossover lines.

The calculated hyperfine splittings, and their differences from the observed values of the $R(56) 32-0$ transition, are listed in Table 1 . The standard deviation is $\sim 500 \mathrm{~Hz}$ for the fit that includes only main lines. The main lines of the $R(56) 32-0$ transition were also measured and calculated by Arie and Byer. ${ }^{10}$ We achieved an uncertainty more than tenfold smaller in both the measured and the fitted results than in the results reported in Ref. 10. Fifteen crossover lines are included in the calculation for the $R(56) 32-0$ transition. The standard deviation of the fit, including both the main and the crossover lines, is $\sim 0.95 \mathrm{kHz}$. The fitted hyperfine constants for both the upper and the lower levels of the $R$ (56)32-0 transition are listed in Table 3 .

For the $P$ (54)32-0 transition, the calculated hyperfine splittings and their differences from the observed values are listed in Table 2. The standard deviation is $\sim 400 \mathrm{~Hz}$ for the fit that includes only main lines. Eight crossover lines are included in the calculation for the $P(54) 32-0$ transition. The standard deviation of the fit, including both the main and the crossover lines, is $\sim 1.13 \mathrm{kHz}$. The fitted hyperfine constants for both the upper and the lower levels of the $P(54) 32-0$ transition are also listed in Table 3.

The uncertainty in the absolute values of the hyperfine constants listed in Table 3 comes mainly from the measurement uncertainty of the crossover lines, which is due to their smaller $\mathrm{S} / \mathrm{N}$ ratio. The recoil shift of the saturation resonances is not included in the present calculation. A detailed discussion of the intensities and recoil structure of the main components and the crossovers can be found in Ref. 32 .

We also list the relative values of the hyperfine constants $\Delta(e Q q), \Delta C, \Delta d$, and $\Delta \delta$ in Table 3 . The calculated $\Delta(e Q q) \quad(=1908.432 \mathrm{MHz})$ for the $P(54) 32-0$ transition is in complete coincidence with the value [=1908.432(1) MHz] in Ref. 13, where only the main lines have been included. This is to say that at this level of uncertainty (several hundreds of hertz for both the observation and the calculation), the main hyperfine lines can still be well described by the differences in the hyperfine constants. Empirical formulas for $\Delta(e Q q), \Delta C, \Delta d$, and $\Delta \delta$ were discussed in Ref. 33. The calculated $\Delta(e Q q)$ [=1908.381(1) MHz] for the $R(56) 32-0$ transition is smaller but more accurate than the value $[=1908.406(10)$ $\mathrm{MHz}]$ in Ref. 11.

By combining the calculated ground state $e Q q^{\prime \prime}\left(J^{\prime \prime}\right.$ $=54,56)$ with the $e Q q^{\prime \prime}\left(J^{\prime \prime}=13,15\right)$ obtained in Ref. 21 , we found a formula for the rotational dependence of $e Q q^{\prime \prime}$. As listed in Table 3, our calculated $e Q q^{\prime \prime}$ shows a difference between $J^{\prime \prime}=54$ and $J^{\prime \prime}=56$ that is larger than that of Bordé and co-workers. We list $e Q q^{\prime \prime}$ values for the $R$ (15)43-0 and $P(13) 43-0$ transitions $^{21}$ in Table 3 for comparison. Figure 3 shows the hyperfine constant 


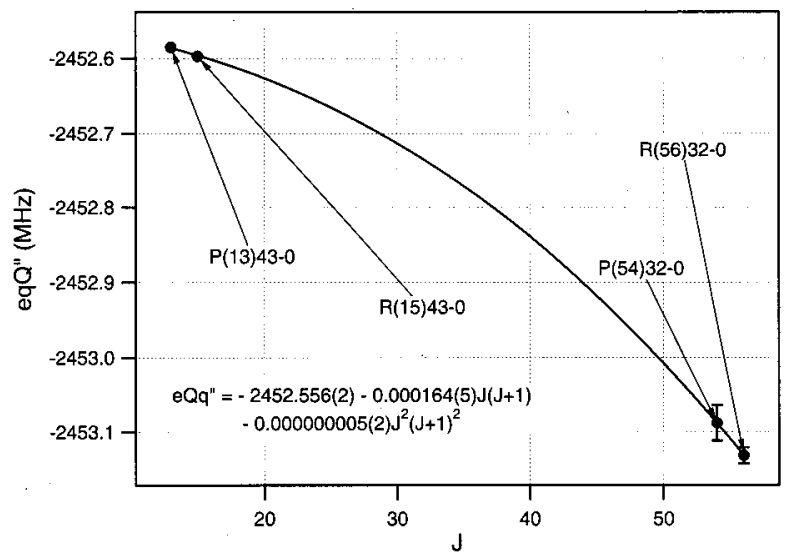

Fig. 3. Ground-state electric quadrupole hyperfine constant $e Q q^{\prime \prime}$ as a function of rotational quantum number $J$. Filled circles, $e Q q^{\prime \prime}$ values calculated from the observed hyperfine splittings. Solid curve, the fitting result for both $J(J+1)$ and $J^{2}(J+1)^{2}$ terms. The $e Q q^{\prime \prime}$ values of $P(13) 43-0$ and $R(15) 43-0$ transitions are taken from Ref. 21.

$e Q q^{\prime \prime}$ as a function of the rotational quantum number $J$. We propose the following formula:

$$
e Q q^{\prime \prime}=e Q q_{0}^{\prime \prime}+\alpha J(J+1)+\beta J^{2}(J+1)^{2},
$$

to express the $J$ dependence of ground state $\left(v^{\prime \prime}\right.$ $=0) e Q q^{\prime \prime}$, where $e Q q_{0}^{\prime \prime}$ is ground state $e Q q^{\prime \prime}$ without any vibrational and rotational effects and $\alpha$ and $\beta$ are the coefficients of the rotational effect. By fitting the calculated $e Q q^{\prime \prime}$ data to the proposed formula, we obtained

$$
\begin{aligned}
e Q q_{0}^{\prime \prime} & =-2452.556(2) \mathrm{MHz} \\
\alpha & =-0.164(5) \mathrm{kHz} \\
\beta & =-0.005(2) \mathrm{Hz} .
\end{aligned}
$$

The fitting result is shown in Fig. 3 as a solid curve. We obtained a smaller chi-square value $\left(2.2 \times 10^{-6}\right)$ of the fitting, by using the formula including both the $J(J$ $+1)$ and the $J^{2}(J+1)^{2}$ terms, than that $\left(8.1 \times 10^{-6}\right)$ of the fitting obtained by using the formula including only the $J(J+1)$ term.

The knowledge obtained of the ground-state hyperfine constants is important in metrology, because one can then reliably calculate the influence from neighboring weak transitions on the main lines that may be used for frequency references.

\section{DISCUSSION}

The obtained rotation dependence of the ground state $e Q q^{\prime \prime}$ is naturally considered to be associated with the centrifugal distortion effect in the electric quadrupole hyperfine interaction. The centrifugal force changes the distance between the two nuclei in the molecule, which changes the value of the electric field gradient $q$. A simple analogy exists between the calculation of rotational energy and the present $J$ dependence of $e Q q^{\prime \prime}$. As shown in the first term of Eq. (3), the rotational energy for a rigid rotor is expressed as

$$
E(J)=B J(J+1) .
$$

The largest correction for this expression is the centrifugal distortion correction shown in the second term of Eq. (3). Thus the rotational energy with centrifugal distortion correction is expressed as

$$
E(J)=B J(J+1)-D J^{2}(J+1)^{2} .
$$

At $J \sim 55$, the third term of Eq. (3) is only $0.03 \%$ of the $D$-dependent term. Equation (8) can also be written as

$$
E(J)=B^{\prime} J(J+1),
$$

where

$$
B^{\prime}=B-D J(J+1)
$$

is the centrifugal-distortion-corrected rotational constant (and we note that both $B$ and $D$ are positive). Equation (11) is analogous to the first two terms of Eq. (4), which we have proposed to represent the electric quadrupole hyperfine constant.

To illustrate the issue, mechanically we have

$$
r_{e}=\left(\frac{h}{4 \pi^{2} B M}\right)^{1 / 2},
$$

where $r_{e}$ and $M$ are the equilibrium separation and the mass of the iodine nuclei, respectively. Equations (11) and (12) allow us to estimate the change of $r_{e}, \Delta r_{e}$, with $J$ as

$$
\Delta r_{e} / r_{e}=1 / 2(D / B) J(J+1),
$$

$\sim+3.7 \times 10^{-4}$ at $J \sim 55$. We note that, on the one hand, our experiment associates an increase in $|e Q q|$ with increasing $J$ and $r_{e}$, beginning from $\left|e Q q_{0}\right|$ $=2452.556(2) \mathrm{MHz}$ for the ground electronic state with $v^{\prime \prime}=0$ and $J^{\prime \prime}=0$, as shown in Fig. 3 and Eq. (5). On the other hand, stretching of the molecule with increasing $J$ leads to the separated-atom picture, with the limiting atomic $\left|e Q q_{A}\right|=2292.71 \mathrm{MHz} .{ }^{34}$ This nonmonotonic behavior of $e Q q\left(r_{e}\right)$ will be fascinating to explore in the future.

More-detailed theoretical discussions can be found in Refs. 35-37. In general, the magnetic and electric fields inside a molecule depend on the internuclear distances and hence on the vibration-rotation state of the molecule. Any molecular constant associated with a given hyperfine interaction can then be considered a function of the internuclear distance and expressed as a Taylor expansion about the equilibrium position. For a diatomic molecule in the electronic state ${ }^{1} \Sigma$, the average value in the vibration-rotation state $|v J\rangle$ of an operator $X$, a function of internuclear distance $r$, can be expanded in terms of the relative distance $\xi$ to the equilibrium position $r_{e}$ (in the present case $X$ is a short notation for $e Q q$ ):

$$
\langle X\rangle_{v J}=X_{e}+X_{e}^{\prime}\langle\xi\rangle_{v J}+(1 / 2) X_{e}^{\prime \prime}\left\langle\xi^{2}\right\rangle_{v J}+\ldots,
$$

where $\xi=\left(R-R_{e}\right) / R_{e}$ and $\langle X\rangle_{v J}=\langle v J|X| v J\rangle$.

The various matrix elements $\left\langle\xi^{n}\right\rangle_{v J}=\left\langle v J\left|\xi^{n}\right| v J\right\rangle$ can be expressed as power series in $v+1 / 2$ and $J(J+1)$ :

$$
\left\langle\xi^{n}\right\rangle_{v J}=\sum_{l j} Z_{l j}^{(n)}[v+(1 / 2)]^{l}[J(J+1)]^{j},
$$

where the coefficients $Z_{l j}^{(n)}$ are functions of $B_{e} / \omega_{e}$ and of Dunham's potential constants $a_{i}$. 
A calculation of the coefficient $Z_{l j}^{(n)}$ valid to third order in $B_{e} / \omega_{e}$ was made by Herman and Short. ${ }^{35}$ If we limit ourselves to second order in $B_{e} / \omega_{e}$,

$$
\begin{aligned}
\langle X\rangle_{v J}= & X_{e}+\frac{B_{e}}{\omega_{e}}\left(v+\frac{1}{2}\right)\left(X_{e}^{\prime \prime}-3 a_{1} X_{e}^{\prime}\right) \\
& +\left(v+\frac{1}{2}\right)^{2}\left(\frac{B_{e}}{\omega_{e}}\right)^{2} \\
& \times\left[X_{e}^{\prime}\left(-15 a_{3}+39 a_{1} a_{2}-\frac{45}{2} a_{1}^{3}\right)\right. \\
& \left.+\frac{1}{2} X_{e}^{\prime \prime}\left(15 a_{1}^{2}-6 a_{2}\right)-\frac{15}{6} X_{e}^{\prime \prime \prime} a_{1}+\frac{1}{4} X_{e}^{\prime \prime \prime \prime}\right] \\
& +4\left(\frac{B_{e}}{\omega_{e}}\right)^{2} X_{e}^{\prime} J(J+1),
\end{aligned}
$$

in agreement with the formula of de Leeuw and Dymanus $^{36}$ and of Watson. ${ }^{37,38}$ The last term is of course the interesting one to us because it provides the quantum-mechanical justification of the simple formula derived in the present paper from semiclassical considerations with the a priori knowledge that $D_{e} / B_{e}$ $=4\left(B_{e} / \omega_{e}\right)^{2}$. As a result, the variation with $J$ of $e Q q$ for diatomic molecules is expressed as

$$
e Q q=e Q q_{0}+4\left(B_{e} / \omega_{e}\right)^{2} J^{\prime \prime}\left(J^{\prime \prime}+1\right)(e Q q)^{\prime}+\ldots .
$$

At the next order in $B_{e} / \omega_{e}$ we find a crossed term in $(v+1 / 2) J(J+1)$ that has to be considered in the future:

$$
\begin{aligned}
2\left(\frac{B_{e}}{\omega_{e}}\right)^{3} & {\left[3\left(8+9 a_{1}+9 a_{1}^{2}-8 a_{2}\right) X_{e}^{\prime}\right.} \\
& \left.-3\left(1+3 a_{1}\right) X_{e}^{\prime \prime}+2 X_{e}^{\prime \prime \prime}\right]\left(v+\frac{1}{2}\right) J(J+1) .
\end{aligned}
$$

The coefficients $B_{e}, \omega_{e}, a_{1}, a_{2}, \ldots$ are known from vibration-rotation data.

More interestingly, in the near future a larger number of accurate data will enable one to invert the problem so that one can find the functional dependence of $e Q q(r)$ on the nucleus separation for different vibration and rotation states. ${ }^{37-39}$ With that information we should be able to start exploration of the molecular bonding characteristics.

The rotational dependence of $e Q q$ has also been observed for ${ }^{189} \mathrm{OsO}_{4}$, which is a spherical-topped molecule. ${ }^{40}$ The $J$ dependence of $e Q q$ was visualized as a parabola and confirmed to be affected predominantly by centrifugal distortion. The vibrational and Coriolis-type contributions to the $J$ dependence were also discussed. In ${ }^{189} \mathrm{OsO}_{4}$, the tetrahedral symmetry of the molecule imposes a null electric field gradient at the osmium location. Hence, as expected, $e Q q^{\prime \prime} \approx 0$ was obtained for the ground vibrational level with $J=0$.

With Eqs. (4)-(7) we can calculate $e Q q^{\prime \prime}$ precisely for all the $J$ levels in the ground state with $v^{\prime \prime}=0$. For $J^{\prime \prime}$ $=57, e Q q^{\prime \prime}$ is calculated to be $-2453.153 \mathrm{MHz} . \quad \Delta e Q q$ for the $R$ (57)32-0 transition was determined ${ }^{13}$ to be 1908.381 MHz. Therefore we obtain the upper level
Table 4. Calculated Electric Quadrupole Hyperfine Constants of the $B$ state $\left(v^{\prime}=32\right)$

\begin{tabular}{lc}
\hline$J^{\prime}$ & $e Q q^{\prime}(\mathrm{MHz})$ \\
\hline 53 & -544.656 \\
57 & -544.751 \\
58 & -544.772 \\
\hline
\end{tabular}

$e Q q^{\prime}$ for the $R$ (57)32-0 transition as $-544.772 \mathrm{MHz}$. In Table 4 , three calculated $e Q q^{\prime}$ are listed. Rotational dependence is again observed for the upper level $e Q q^{\prime}$, with approximately the same coefficient. In the excited states, the electric field gradient is different from that in the ground states, and the vibration of the molecule may also play a role.

Ground state $e Q q^{\prime \prime}$ of $\mathrm{I}_{2}$ was previously investigated for $J^{\prime \prime}=0-10 \quad\left(v^{\prime \prime}=1\right) \quad$ by molecular-beam laser spectroscopy. ${ }^{41}$ No rotational dependence was found because of the large measurement uncertainty of $e Q q^{\prime \prime}$ $(\sim 4.5 \mathrm{MHz})$. The rotational dependence of $e Q q^{\prime \prime}$ and $\Delta e Q q$ was discussed by Špirko and Blabla ${ }^{42}$ and by Knöckel et al. ${ }^{43}$ respectively; however, knowledge of their values was again limited by measurement uncertainty. With the high-frequency stability of our laser systems that results from the high $\mathrm{S} / \mathrm{N}$ ratio and narrow linewidth of the observed signal, we have reduced the uncertainty of $e Q q^{\prime \prime}$ to $\sim 10 \mathrm{kHz}$.

With the high stability available from our lasers, we can also investigate the characteristics of the laser systems more precisely. For example, in the international comparison of the $\mathrm{I}_{2}$-stabilized Nd:YAG lasers, the hyperfine splitting was found to vary slightly between two different systems. ${ }^{18}$ We believe that the difference in wavefront curvature and the residual amplitude modulation in the phase modulation process are the main contributions to the difference of the hyperfine splittings. Another contribution may come from an influence of recoil splitting. In fact, we did perform another least-squares fit with the measured hyperfine splittings properly modified to reflect the estimated recoil shifts associated with the main lines and the crossovers. Results of calculations with the recoil corrections included indicate that the fit uncertainties remain similar. The variation of $e Q q^{\prime \prime}$ owing to the recoil correction is negligible, well within the standard deviation of the fit. However, the value of $C^{\prime \prime}$ does change, depending on whether the recoil correction is included, with a variation of the order of 2-5 times the fit uncertainty. These issues can be investigated more thoroughly when the stability and reliability of the laser systems are improved further. Also, study of the transitions of lower rotational quantum numbers will offer a more-precise determination of the $e Q q$ value owing to the stronger intensities of the crossover transitions. Success in these directions will allow us to achieve an even smaller measurement uncertainty in the hyperfine splittings and to test the validity of the four-term Hamiltonian with higher accuracy.

\section{ACKNOWLEDGMENTS}

The research at JILA was supported in part by the U.S. Air Force Office of Scientific Research, the National Sci- 
ence Foundation, and the National Institute of Standards and Technology as part of its program of research in precision measurements and advanced frequency standards. F.-L. Hong appreciates support by the National Research Laboratory of Metrology, the Ministry of International Trade and Industry, and the Science and Technology Agency, Japan, for his overseas study. Ch. J. Bordé is especially grateful to his good friend J. L. Hall for the kind invitation to work during summer 1998 in Hall's laboratory at JILA; Bordé thanks Jacques Vigué for many enlightening discussions. Ye's e-mail address is ye@jila.colorado.edu.

*Permanent address, National Research Laboratory of Metrology, Tsukuba, Ibaraki 305-8536, Japan.

**Staff member, Quantum Physics Division, National Institute of Standards and Technology.

${ }^{\dagger}$ Permanent address, Laboratory for Quantum Optics, East China Normal University, Shanghai, China.

${ }^{\ddagger}$ Bureau International des Poids et Measures, Sèvres Cedex, France.

IPermanent address, Laboratoire de Physique des Lasers, Université Paris-Nord, Avenue J.-B. Clément, 93430 Villetaneuse, France.

\section{REFERENCES}

1. M. D. Levenson and A. L. Schawlow, "Hyperfine interactions in molecular iodine," Phys. Rev. A 6, 10-20 (1972).

2. H. J. Foth and F. Spieweck, "Hyperfine Structure of the $R(98)$, 58-1 line of ${ }^{127} \mathrm{I}_{2}$ at $514.5 \mathrm{~nm}$," Chem. Phys. Lett. 65, 347-352 (1979).

3. Ch. J. Bordé, G. Camy, B. Decomps, J.-P. Descoubes, and J. Vigué, "High precision saturation spectroscopy of ${ }^{127} \mathrm{I}_{2}$ with argon lasers at $5145 \AA$ and $5017 \AA$. I. Main resonances," J. Phys. (Paris) 42, 1393-1411 (1981).

4. G. R. Hanes and C. E. Dahlstrom, "Iodine hyperfine structure observed in saturated absorption at $633 \mathrm{~nm}$," Appl. Phys. Lett. 14, 362-364 (1969).

5. P. Cérez and S. J. Bennett, "Helium-neon laser stabilized by saturated absorption in iodine at $612 \mathrm{~nm}$," Appl. Opt. 18, 1079-1083 (1979).

6. J.-M. Chartier, S. Fredin-Picard, and L. Robertsson, "Frequency-stabilized $543 \mathrm{~nm} \mathrm{HeNe}$ laser system: a new candidate for the realization of the meter?" Opt. Commun. 74, 87-92 (1989)

7. B. Couillaud and A. Ducasse, "Saturated absorption experiments using a free running cw dye laser," Opt. Commun. 13, 398-401 (1975).

8. A. Razet and S. Picard, "A tabulation of calculations of the hyperfine structure in ${ }^{127} \mathrm{I}_{2}$," Metrologia 33, 19-27 (1996).

9. J. L. Hall, L.-S. Ma, M. Taubman, B. Tiemann, F.-L. Hong, O. Pfister, and J. Ye, "Stabilization and frequency measurement of the $\mathrm{I}_{2}$-stabilized Nd:YAG laser," IEEE Trans. Instrum. Meas. 48, 583-586 (1999).

10. A. Arie and R. L. Byer, "Laser heterodyne spectroscopy of ${ }^{127} \mathrm{I}_{2}$ hyperfine structure near $532 \mathrm{~nm}$," J. Opt. Soc. Am. B 10, 1990-1997 (1993).

11. A. Arie and R. L. Byer, "The hyperfine structure of the ${ }^{127} \mathrm{I}_{2}$ $P(119)$ 35-0 transition," Opt. Commun. 111, 253-258 (1994).

12. M. L. Eickhoff and J. L. Hall, "Optical frequency standard at $532 \mathrm{~nm}$," IEEE Trans. Instrum. Meas. 44, 155-158 (1995).

13. J. Ye, L. Robertsson, S. Picard, L.-S. Ma, and J. L. Hall, "Absolute frequency atlas of molecular $\mathrm{I}_{2}$ lines at $532 \mathrm{~nm}$," IEEE Trans. Instrum. Meas. 48, 544-549 (1999).
14. T. J. Quinn, "Practical realization of the definition of the metre (1997)," Metrologia 36, 211-244 (1999).

15. P. A. Jungner, S. Swartz, M. Eickhoff, J. Ye, J. L. Hall, and S. Waltman, "Absolute frequency of the molecular iodine transition $R$ (56)32-0 near 532 nm," IEEE Trans. Instrum. Meas. 44, 151-154 (1995).

16. P. Jungner, M. L. Eickhoff, S. D. Swartz, J. Ye, and J. L. Hall, "Stability and absolute frequency of molecular iodine transitions near $532 \mathrm{~nm}$," in Laser Frequency Stabilization and Noise Reduction, Y. Shevy, ed., Proc. SPIE 2378, 22-34 (1995).

17. F.-L. Hong, J. Ishikawa, T. H. Yoon, L.-S. Ma, J. Ye, and J. L. Hall, "A portable $\mathrm{I}_{2}$-stabilized Nd:YAG laser for wavelength standards at $532 \mathrm{~nm}$ and $1064 \mathrm{~nm}$," in Recent Developments in Optical Gauge Block Metrology, N. Brown and J. E. Decker, eds., Proc. SPIE 3477, 2-10 (1998).

18. F.-L. Hong, J. Ishikawa, J. Yoda, J. Ye, L.-S. Ma, and J. L. Hall, "Frequency comparison of ${ }^{127} \mathrm{I}_{2}$-stabilized Nd:YAG lasers," IEEE Trans. Instrum. Meas. 48, 532-536 (1999).

19. M. S. Sorem, T. W. Hänsch, and A. L. Schawlow, "Nuclear quadrupole coupling in the ${ }^{1} \Sigma_{g}{ }^{+}$and ${ }^{3} \Pi_{o u}{ }^{+}$states of molecular iodine," Chem. Phys. Lett. 17, 300-302 (1972).

20. A. Yokozeki and J. S. Muenter, "Laser fluorescence state selected and detected molecular beam magnetic resonance in I, , J. Chem. Phys. 72, 3796-3804 (1980).

21. J.-P. Wallerand, F. du Burck, B. Mercier, A. N. Goncharov, M. Himbert, and Ch. J. Bordé, "Frequency measurements of hyperfine splittings in ground rovibronic states of $\mathrm{I}_{2}$ by stimulated resonant Raman spectroscopy,” Eur. Phys. J. D 6, 63-76 (1999).

22. (Personal communication of Ch. J. Bordé with J. L. Hall, 1998.)

23. T. W. Hänsch and B. Couillaud, "Laser frequency stabilizaion by polarization spectroscopy of a reflecting reference cavity," Opt. Commun. 35, 441-444 (1980).

24. G. Camy, C. J. Bordé, and M. Ducloy, "Heterodyne saturation spectroscopy through frequency modulation of the saturation beam," Opt. Commun. 41, 325-330 (1982), especially Refs. 6 and 7 therein.

25. J. H. Shirley, "Modulation transfer processes in optical heterodyne saturation spectroscopy," Opt. Lett. 7, 537-539 (1982).

26. L. S. Ma, J. H. Shirley, L. Hollberg, and J. L. Hall, "Modulation transfer spectroscopy for stabilizing lasers," U.S. patent 4,590,597 (May 26, 1986).

27. M. Kroll, "Hyperfine structure in the visible moleculariodine absorption spectrum," Phys. Rev. Lett. 23, 631-633 (1969)

28. P. R. Bunker and G. R. Hanes, "Nuclear spin-spin coupling in the spectrum of $\mathrm{I}_{2}$ at $6328 \AA$," Chem. Phys. Lett. 28, 377379 (1974).

29. L. A. Hackel, K. H. Casleton, S. G. Kukolich, and S. Ezekiel, "Observation of magnetic octupole and scalar spin-spin interaction in $\mathrm{I}_{2}$ using laser spectroscopy," Phys. Rev. Lett. 35, 568-571 (1975).

30. See also B. M. Landsberg, "Nuclear hyperfine splittings in $B-X$ electronic band system of ${ }^{127} \mathrm{I}_{2}$," Chem. Phys. Lett. 43, 102-103 (1976).

31. S. Gerstenkorn and P. Luc, "Description of the absorption spectrum of iodine recorded by means of Fourier transform spectroscopy: the $(B-X)$ system," J. Phys. (Paris) 46, 867-881 (1985).

32. Ch. J. Bordé, G. Camy, N. and B. Decomps, "Measurement of the recoil shift of saturation resonances of ${ }^{127} \mathrm{I}_{2}$ at $5145 \AA$ : a test of accuracy for high-resolution spectroscopy," Phys. Rev. A 20, 254-268 (1979). J. Bordeé and Ch. J. Bordé, "Intensities of hyperfine components in saturation spectroscopy," J. Mol. Spectrosc. 78, 353-378 (1979).

33. A. Razet and S. Picard, "A test of new empirical formulas for the prediction of hyperfine component frequencies in ${ }^{127} \mathrm{I}_{2}$," Metrologia 34, 181-186 (1997).

34. V. Jaccarino, J. G. King, R. A. Satten, and H. H. Stroke, "Hyperfine structure of $\mathrm{I}^{127}$. Nuclear magnetic octupole moment," Phys. Rev. 94, 1798-1616 (1954). 
35. R. M. Herman and S. Short, "New theoretical method for the accurate calculation of expectation values on functions of internuclear in ${ }^{1} \Sigma$-state diatomic molecules," J. Chem. Phys. 48, 1266-1272 (1968).

36. F. H. de Leeuw and A. Dymanus, "Magnetic properties and molecular quadrupole moment of $\mathrm{HF}$ and $\mathrm{HCl}$ by molecular-beam electric-resonance spectroscopy," J. Mol. Spectrosc. 48, 427-445 (1973).

37. J. K. G. Watson, "The inversion of diatomic vibrationrotation expectation values," J. Mol. Spectrosc. 74, 319-321 (1979).

38. See also P. R. Bunker, "The breakdown of the BornOppenheimer approximation for a diatomic molecule: the dipole moment and nuclear quadrupole coupling constants," J. Mol. Spectrosc. 45, 151-158 (1973).

39. G. Gouédard, N. Billy, B. Girard, and J. Vigué, "Hyperfine structure measurements in the IF $B-X$ system," J. Phys. II 2, 813-825 (1992)

40. Ch. Chardonnet, M. L. Palma, and Ch. J. Bordé, "Hyperfine interactions in the $v_{3}$ band of osmium tetroxide: the electric quadrupole interaction in ${ }^{189} \mathrm{OsO}_{4}, " \mathrm{~J}$. Mol. Spectrosc. 170, 542-566 (1995).

41. M. Wakasugi, T. Horiguchi, M. Koizumi, and Y. Yoshizawa, "Hyperfine structure near the 13-1 band head in the $B-X$ transition of ${ }^{127} \mathrm{I}_{2}$," J. Opt. Soc. Am. B 5, 2298-2304 (1988).

42. V. Spirko and J. Blabla, "Nuclear quadrupole coupling functions of the ${ }^{1} \Sigma_{\sigma}{ }^{+}$and ${ }^{3} \Pi_{0 u}{ }^{+}$states of molecular iodine," J. Mol. Spectrosc. 129, 59-71 (1988).

43. H. Knöckel, S. Kremser, B. Bodermann, and E. Tiemann, "High precision measurement of hyperfine structures near $790 \mathrm{~nm}$ of $\mathrm{I}_{2}$, Z. Phys. D 37, 43-48 (1996). 